\title{
ON A PROBLEM OF SIDON FOR POLYNOMIALS OVER FINITE FIELDS
}

\author{
WENTANG KUO AND SHUNTARO YAMAGISHI
}

\begin{abstract}
Let $\omega$ be a sequence of positive integers. Given a positive integer $n$, we define
\end{abstract}

$$
r_{n}(\omega)=|\{(a, b) \in \mathbb{N} \times \mathbb{N}: a, b \in \omega, a+b=n, 0<a<b\}| .
$$

S. Sidon conjectured that there exists a sequence $\omega$ such that $r_{n}(\omega)>0$ for all $n$ sufficiently large and, for all $\epsilon>0$,

$$
\lim _{n \rightarrow \infty} \frac{r_{n}(\omega)}{n^{\epsilon}}=0
$$

P. Erdős proved this conjecture by showing the existence of a sequence $\omega$ of positive integers such that

$$
\log n \ll r_{n}(\omega) \ll \log n .
$$

In this paper, we prove an analogue of this conjecture in $\mathbb{F}_{q}[T]$, where $\mathbb{F}_{q}$ is a finite field of $q$ elements. More precisely, let $\omega$ be a sequence in $\mathbb{F}_{q}[T]$. Given a polynomial $h \in \mathbb{F}_{q}[T]$, we define

$$
r_{h}(\omega)=\left|\left\{(f, g) \in \mathbb{F}_{q}[T] \times \mathbb{F}_{q}[T]: f, g \in \omega, f+g=h, \operatorname{deg} f, \operatorname{deg} g \leq \operatorname{deg} h, f \neq g\right\}\right| .
$$

We show that there exists a sequence $\omega$ of polynomials in $\mathbb{F}_{q}[T]$ such that

$$
\operatorname{deg} h \ll r_{h}(\omega) \ll \operatorname{deg} h
$$

for $\operatorname{deg} h$ sufficiently large.

\section{INTRODUCTION}

In the course of investigations on Fourier series by S. Sidon, several questions arose concerning the existence and nature of certain positive integer sequences $\omega$ for which $r_{n}(\omega)=\mid\{(a, b) \in$ $\mathbb{N} \times \mathbb{N}: a, b \in \omega, a+b=n, 0<a<b\} \mid$ is bounded or, in some sense, exceptionally small, where $|S|$ denotes the cardinality of the set $S$. In particular, he asked the following question in 1932, known as the Sidon Problem [1]:

Does there exist a sequence $\omega$ such that $r_{n}(\omega)>0$ for all $n$ sufficiently large and, for all $\epsilon>0$,

$$
\lim _{n \rightarrow \infty} \frac{r_{n}(\omega)}{n^{\epsilon}}=0 ?
$$

In 1954, P. Erdős answered positively to the question by proving the following [1]:

Theorem (Erdős). There exists a sequence $\omega$ such that

$$
\log n \ll r_{n}(\omega) \ll \log n
$$

for all $n$ sufficiently large.

In other words, there exists a "thin" set $\omega$ such that every positive integer sufficiently large can be represented as a sum of two elements in $\omega$. On the other direction, Erdős and Rényi proved the following theorem in [2] that there exists a "thick" set $\omega$ such that $r_{n}(\omega)$ is bounded for all $n$.

Date: September 5, 2021.

2010 Mathematics Subject Classification. 11K31, 11B83, $11 \mathrm{~T} 55$.

Key words and phrases. Sidon sets, Probabilistic number theory.

The research of the first author was supported by an NSERC discovery grant. 
Theorem (Erdős-Rényi). For any $\varepsilon>0$, there exists a positive number $G=G(\varepsilon)$ and a sequence $\omega$, such that $r_{n}(\omega)<G$ for all $n$ and

$$
|\{m \in \omega: m \leq n\}|>n^{\frac{1}{2}-\varepsilon}
$$

for sufficiently large $n$.

We note that the result is best possible up to the $\varepsilon$ term. One way to see this fact is by the pigeon hole principle. Suppose we have $\omega_{0} \subseteq \mathbb{N}$, where $r_{n}\left(\omega_{0}\right)<G$ for all $n \in \mathbb{N}$. Given any $m_{1}, m_{2} \in\left\{m \in \omega_{0}: m \leq n\right\}$, we have $1<m_{1}+m_{2} \leq 2 n$. Therefore, by the pigeon hold principle, it follows that

$$
G>\max _{1<m \leq 2 n} r_{m}\left(\omega_{0}\right) \geq \frac{\left|\left\{m \in \omega_{0}: m \leq n\right\}\right|^{2}-\left|\left\{m \in \omega_{0}: m \leq n\right\}\right|}{2(2 n-1)} .
$$

Consequently, we obtain

$$
\left|\left\{m \in \omega_{0}: m \leq n\right\}\right| \ll n^{1 / 2} .
$$

In this paper, we prove an analogue of these results in the setting of $\mathbb{F}_{q}[T]$.

Let $\omega$ be a sequence of polynomials in $\mathbb{F}_{q}[T]$. For each $h \in \mathbb{F}_{q}[T]$, we define

$$
r_{h}(\omega)=\left|\left\{(f, g) \in \mathbb{F}_{q}[T] \times \mathbb{F}_{q}[T]: f, g \in \omega, h=f+g, \operatorname{deg} f, \operatorname{deg} g \leq \operatorname{deg} h, f \neq g\right\}\right| .
$$

Note $\operatorname{deg} f$ is the degree of $f \in \mathbb{F}_{q}[T]$ with the convention that $\operatorname{deg} 0=-\infty$. We prove the following results.

Theorem 1. There exists a sequence $\omega$ of polynomials in $\mathbb{F}_{q}[T]$ such that

$$
\operatorname{deg} h \ll r_{h}(\omega) \ll \operatorname{deg} h
$$

for $\operatorname{deg} h$ sufficiently large.

On the other direction, we prove that there exists a "thick" set with bounded value $r_{h}(\omega)$. We denote the elements of $\omega$ by $\omega=\left\{f_{i}\right\}_{i \in \mathbb{N}}$, where $\operatorname{deg} f_{i} \leq \operatorname{deg} f_{j}(i<j)$.

Theorem 2. For each $\epsilon>0$, there exists a sequence $\omega=\left\{f_{i}\right\}$ of polynomials in $\mathbb{F}_{q}[T]$ and a positive integer $K$ such that $r_{h}(\omega)<K$ for all $h \in \mathbb{F}_{q}[T]$ and $q^{\operatorname{deg} f_{i}} \ll i^{2+\epsilon}$.

For each $h \in \mathbb{F}_{q}[T]$, we define

$$
t_{h}(\omega)=\left|\left\{(f, g) \in \mathbb{F}_{q}[T] \times \mathbb{F}_{q}[T]: f, g \in \omega, h=f-g, \operatorname{deg} f, \operatorname{deg} g \leq \operatorname{deg} h\right\}\right| .
$$

We also prove the following variation of the existence of thick sets.

Theorem 3. For each $\epsilon>0$, there exists a sequence $\omega=\left\{f_{i}\right\}$ of polynomials in $\mathbb{F}_{q}[T]$ and a positive integer $K^{\prime}$ such that $t_{h}(\omega)<K^{\prime}$ for all $h \in \mathbb{F}_{q}[T]$ and $q^{\operatorname{deg} f_{i}} \ll i^{2+\epsilon}$.

We prove our theorems following the methods of Chapter III of [3], which utilizes the language of probability. Roughly speaking, we set up a probability space to study the probability of the events $\left\{\omega \mid r_{h}(\omega)=d\right\}$ for all non-negative integer $d$. Using the Borel-Cantelli lemma, we show that the sequences satisfy the desired properties with probability 1 . We also remark that Theorems 2 and 3 have been generalized to $m$-fold sums and differences by K. E. Hare and the second author in [4].

The organization of this paper is as follows. In Section 2, we first review the basic probability theory and state the Borel-Cantelli lemma. Next, in Section 3, we state the equivalent statements of our theorems and set up the probability space used in our proof. In Section 4, we establish several technical lemmas. Finally, the remaining sections are devoted to the proof of our main results. 


\section{Preliminaries}

We start with probability theory. Let $\left\{X_{j}\right\}$ be a sequence of spaces and write

$$
X=\prod_{j=0}^{\infty} X_{j} .
$$

Let $\mathcal{M}_{j}$ be a $\sigma$-algebra of subsets of $X_{j}$. A measurable rectangle with respect to the sequence $\left\{\mathcal{M}_{j}\right\}$ is defined to be a subset $W$ of $X$ which is representable in the form

$$
W=\prod_{j=0}^{\infty} W_{j},
$$

where $W_{j} \in \mathcal{M}_{j}$ and $W_{j}=X_{j}$ except for finitely many $j$. The following two theorems are standard results in probability theory, see for example [3, p. 123, Thm. 5] and [3, p. 135] for reference.

Theorem 4. [3, p. 123, Theorem 5] Let $\left\{\left(X_{j}, \mathcal{M}_{j}, P_{j}\right)\right\}_{j \geq 0}$ be a sequence of probability spaces, and write

$$
X=\prod_{j=0}^{\infty} X_{j} .
$$

Let $\mathcal{M}$ be the minimal $\sigma$-algebra of subsets of $X$ containing every measurable rectangle with respect to the sequence $\left\{\mathcal{M}_{j}\right\}$. Then there exists a unique measure $P$ on $\mathcal{M}$ with the property that for every non-empty measurable rectangle $W$,

$$
P(W)=\prod_{j=0}^{\infty} P_{j}\left(W_{j}\right),
$$

where the $W_{j}$ are defined by $W=\prod_{j=0}^{\infty} W_{j}, W_{j} \in \mathcal{M}_{j}(j \geq 0)$.

We remark that the product in (5) is, in essence, a finite product by the definition of measurable rectangles with respect to the sequence $\left\{\mathcal{M}_{j}\right\}$. Furthermore, since

$$
P(X)=\prod_{j=0}^{\infty} P_{j}\left(X_{j}\right)=1,
$$

the $\sigma$-algebra $\mathcal{M}$ in conjunction with the measure $P$ constitutes a probability space $(X, \mathcal{M}, P)$.

Theorem 6. [3, p. 135, The Borel-Cantelli Lemma] Let $\left(X^{\prime}, \mathcal{M}^{\prime}, P^{\prime}\right)$ be a probability space. Let $\left\{W_{\ell}\right\}$ be a sequence of measurable events. If

$$
\sum_{\ell=1}^{\infty} P^{\prime}\left(W_{\ell}\right)<\infty,
$$

then, with probability 1 , at most finite number of the events $W_{\ell}$ can occur; or, equivalently,

$$
P^{\prime}\left(\bigcap_{i=1}^{\infty} \bigcup_{\ell=i}^{\infty} W_{\ell}\right)=0 .
$$




\section{Probability Space $(\Omega, \mathcal{M}, P)$}

We let $q=p^{s}$ for a prime $p$, and denote $\mathbb{F}_{q}$ to be the finite field of $q$ elements. Let $\mathbb{F}_{q}[T]$ be the polynomial ring over $\mathbb{F}_{q}$. Let $\iota$ be any bijective map from $\mathbb{Z} \cap[0, q-1]$ to $\mathbb{F}_{q}$. We label each of the polynomials in $\mathbb{F}_{q}[T]$ as follows. Let $\mathbb{Z}_{\geq 0}$ be the set of all non-negative integers. For every $N \in \mathbb{Z}_{>0}$, we define

$$
p_{N}:=\iota\left(c_{0}\right)+\iota\left(c_{1}\right) T+\ldots+\iota\left(c_{n}\right) T^{n},
$$

where $N=c_{0}+c_{1} q+\ldots+c_{n} q^{n}$ and $0 \leq c_{i}<q(1 \leq i \leq n)$. It is clear that this identification gives a one-to-one correspondence of sets between $\mathbb{Z}_{\geq 0}$ and $\mathbb{F}_{q}[T]$.

We use $\omega$ to denote a subsequence of the sequence of all polynomials in $\mathbb{F}_{q}[T]$, i.e. $p_{0}, p_{1}, p_{2}, p_{3}, \ldots$ and $\Omega$ to denote the space of all such sequences $\omega$. By $f \in \omega$, we mean $f \in \mathbb{F}_{q}[T]$ appears in the sequence $\omega$. Given $N \in \mathbb{Z}_{\geq 0}$ and $\omega \in \Omega$, we define

$$
r_{N}(\omega)=\left|\left\{(a, b) \in \mathbb{Z}_{\geq 0} \times \mathbb{Z}_{\geq 0}: p_{a}, p_{b} \in \omega, p_{N}=p_{a}+p_{b}, \operatorname{deg} p_{a}, \operatorname{deg} p_{b} \leq \operatorname{deg} p_{N}, a<b\right\}\right|,
$$

and

$$
t_{N}(\omega)=\left|\left\{(a, b) \in \mathbb{Z}_{\geq 0} \times \mathbb{Z}_{\geq 0}: p_{a}, p_{b} \in \omega, p_{N}=p_{a}-p_{b}, \operatorname{deg} p_{a}, \operatorname{deg} p_{b} \leq \operatorname{deg} p_{N}\right\}\right| .
$$

We prove the following results which our main theorems, namely Theorems 1, 2 and 3, are consequences of.

Theorem 7. There exists a sequence $\omega$ of polynomials in $\mathbb{F}_{q}[T]$ such that

$$
\log N \ll r_{N}(\omega) \ll \log N
$$

for $N$ sufficiently large.

Theorem 8. For each $\epsilon>0$, there exists a sequence $\omega=\left\{p_{b_{j}}\right\}$ of polynomials in $\mathbb{F}_{q}[T]$ and a positive integer $K_{0}$ such that $r_{N}(\omega)<K_{0}$ for all $N \in \mathbb{Z}_{\geq 0}$ and $b_{j} \ll j^{2+\epsilon}$.

Theorem 9. For each $\epsilon>0$, there exists a sequence $\omega=\left\{p_{b_{j}}\right\}$ of polynomials in $\mathbb{F}_{q}[T]$ and a positive integer $K_{0}^{\prime}$ such that $t_{N}(\omega)<K_{0}^{\prime}$ for all $N \in \mathbb{Z}_{\geq 0}$ and $b_{j} \ll j^{2+\epsilon}$.

Since $\operatorname{deg} p_{N} \leq \log _{q} N<\operatorname{deg} p_{N}+1$, we can easily derive Theorems 1, 2, and 3, from Theorems 7,8 , and 9 , respectively.

We now prove the existence of the following probability space. The content of this theorem is essentially [3, p. 141, Theorem 13].

Theorem 10. Let

$$
\alpha_{0}, \alpha_{1}, \alpha_{2}, \alpha_{3}, . .
$$

be real numbers satisfying $0 \leq \alpha_{i} \leq 1(i \geq 0)$. Then there exists a probability space $(\Omega, \mathcal{M}, P)$ with the following two properties:

(i) For every non-negative integer $m$, the event $\mathfrak{B}_{m}=\left\{\omega \in \Omega: p_{m} \in \omega\right\}$ is measurable and $P\left(\mathfrak{B}_{m}\right)=\alpha_{m}$.

(ii) The events $\mathfrak{B}_{0}, \mathfrak{B}_{1}, \mathfrak{B}_{2}, \ldots$ are independent.

Proof. Let $Y$ be the space of two elements, $y_{0}$ and $y_{1}$ say. For each sequence $\omega$ we associate the sequence $\left\{x_{j}\right\}$ of elements of $Y$, defined by

$$
x_{j}= \begin{cases}y_{0}, & \text { if } p_{j} \notin \omega \\ y_{1}, & \text { if } p_{j} \in \omega\end{cases}
$$


for $j \geq 0$. The space $X$ consisting of all the sequences $x=\left\{x_{j}\right\}$ is given by

$$
X=\prod_{j=0}^{\infty} X_{j}
$$

where $X_{j}=Y$ for $j \geq 0$. Let $\mathcal{M}_{j}=\left\{\phi,\left\{y_{0}\right\},\left\{y_{1}\right\}, X_{j}\right\}$, the non-trivial $\sigma$-algebra of $X_{j}$, and let $P_{j}$ be the probability measure on $\mathcal{M}_{j}$ such that $P_{j}\left(\left\{y_{1}\right\}\right)=\alpha_{j}$.

We apply Theorem 4 to the sequence $\left\{X_{j}, \mathcal{M}_{j}, P_{j}\right\}$ of probability spaces. In view of the oneto-one correspondence between the elements of $X$ and $\Omega$, we may denote the resulting probability space as $(\Omega, \mathcal{M}, P)$.

Now, we prove $(\Omega, \mathcal{M}, P)$ satisfies the two properties $(i)$ and $(i i)$. Clearly, we have

$$
\mathfrak{B}_{m}=\left\{\omega \in \Omega: p_{m} \in \omega\right\}=\prod_{j=0}^{\infty} W_{j},
$$

where $W_{j}=X_{j}$ for all $j$ except $j=m$ and $W_{m}=\left\{y_{1}\right\}$. Then, $(i)$ follows, because $\mathfrak{B}_{m} \in \mathcal{M}$ by the definition of $\mathcal{M}$, and by (5) we have

$$
P\left(\mathfrak{B}_{m}\right)=\prod_{j=0}^{\infty} P_{j}\left(W_{j}\right)=P_{m}\left(\left\{y_{1}\right\}\right)=\alpha_{m} .
$$

For $(i i)$, we consider any finite subset of $\left\{\mathfrak{B}_{j}\right\}$, say $\mathfrak{B}_{j_{1}}, \mathfrak{B}_{j_{2}}, \ldots, \mathfrak{B}_{j_{\ell}}$. Then, clearly we have

$$
\bigcap_{i=1}^{\ell} \mathfrak{B}_{j_{i}}=\left\{\omega \in \Omega: p_{j_{i}} \in \omega(1 \leq i \leq \ell)\right\}=\prod_{j=0}^{\infty} W_{j},
$$

where $W_{j}=X_{j}$ for all $j$ except $j=j_{1}, \ldots, j_{\ell}$ and $W_{j_{i}}=\left\{y_{1}\right\}$ for $1 \leq i \leq \ell$. Thus, by (5) and $(i)$ we obtain

$$
P\left(\bigcap_{i=1}^{\ell} \mathfrak{B}_{j_{i}}\right)=\prod_{j=0}^{\infty} P_{j}\left(W_{j}\right)=\prod_{i=1}^{\ell} P_{j_{i}}\left(\left\{y_{1}\right\}\right)=\prod_{i=1}^{\ell} \alpha_{j_{i}}=\prod_{i=1}^{\ell} P\left(\mathfrak{B}_{j_{i}}\right),
$$

from which $(i i)$ follows.

\section{Technical Lemmas}

In this section, we prove several technical lemmas used in our proofs. For each $N \in \mathbb{Z}_{\geq 0}$, let $p_{N} \in \mathbb{F}_{q}[T]$ be as prescribed in the previous section. Define

$$
n:=n(N)=\operatorname{deg} p_{N}=\left\lfloor\log _{q} N\right\rfloor .
$$

Suppose $p \neq 2$. Since $\mathbb{F}_{q}=2 \mathbb{F}_{q}$, we know there exists $p_{N_{0}}$ such that $p_{N}=p_{N_{0}}+p_{N_{0}}$. It is clear that $\operatorname{deg} p_{N_{0}}=n$; therefore, $q^{n} \leq N_{0}<q^{n+1}$. Since $\mathbb{F}_{q}[T]$ is closed under addition, we can uniquely pair up the rest of polynomials of degree less than or equal to $n$ by

$$
p_{N}=p_{a}+p_{\widetilde{a}}
$$

where $a, \widetilde{a} \in \mathbb{Z}_{\geq 0}, a<\widetilde{a}$. We collect all such pairs $(a, \widetilde{a})$ and form

$$
A_{N}=\left\{a \in \mathbb{Z}_{\geq 0}: p_{N}=p_{a}+p_{\widetilde{a}}, a<\widetilde{a}, \text { and } \operatorname{deg} p_{a}, \operatorname{deg} p_{\widetilde{a}} \leq n\right\},
$$

and

$$
\widetilde{A}_{N}=\left\{\widetilde{a} \in \mathbb{Z}_{\geq 0}: p_{N}=p_{a}+p_{\widetilde{a}}, a<\widetilde{a}, \text { and } \operatorname{deg} p_{a}, \operatorname{deg} p_{\widetilde{a}} \leq n\right\}
$$


We have $\left|A_{N}\right|=\left|\widetilde{A}_{N}\right|=\left(q^{n+1}-1\right) / 2$, and

$$
\left\{0, \ldots, q^{n+1}-1\right\}=A_{N} \bigcup \widetilde{A}_{N} \bigcup\left\{N_{0}\right\}
$$

where all the unions are disjoint. Further, it is easy to see that $\left\{0,1, \ldots, q^{n}-1\right\} \subseteq A_{N}$, because if $0 \leq a<q^{n}$, then $p_{a}$ has degree at most $n-1$. Thus, the corresponding $p_{\widetilde{a}}$ must have degree $n$; therefore, $q^{n} \leq \widetilde{a}<q^{n+1}$. Hence, it follows that

$$
\widetilde{A}_{N} \subseteq\left\{q^{n}, q^{n}+1, \ldots, q^{n+1}-1\right\} .
$$

Let $M:=M(N)=\left(q^{n+1}-1\right) / 2$. For convenience we label the $M$ elements of $A_{N}$ by $a_{i}$, where $1 \leq i \leq M$, and the corresponding elements of $\widetilde{A}_{N}$ by $\widetilde{a}_{i}$.

We also define $\lambda_{N}$ and $\lambda_{N}^{\prime}$ to be

$$
\lambda_{N}=\sum_{1 \leq i \leq M} \alpha_{a_{i}} \alpha_{\widetilde{a}_{i}}
$$

and

$$
\lambda_{N}^{\prime}=\sum_{1 \leq i \leq M} \frac{\alpha_{a_{i}} \alpha_{\widetilde{a}_{i}}}{1-\alpha_{a_{i}} \alpha_{\widetilde{a}_{i}}} .
$$

Note when $p=2$, for $N>0$, we do not have to consider the polynomial $p_{N_{0}}$ as above. Thus we let $M:=M(N)=q^{n+1} / 2$ and we can argue in a similar manner.

Define

$$
s_{N}^{*}(\omega)=\sum_{m=0}^{N} \mathbf{1}_{\mathfrak{B}_{m}}(\omega)
$$

where $\mathbf{1}_{\mathfrak{B}_{m}}$ is the characteristic function on the set $\mathfrak{B}_{m}$. Let $E(f)$ denote the expectation of a random variable $f$, defined by $E(f)=\int_{X} f d P$. We define

$$
m_{N}^{*}=E\left(s_{N}^{*}\right)=\sum_{m=0}^{N} \alpha_{m} .
$$

We need our sequence $\left\{\alpha_{j}\right\}$ to satisfy the following condition.

Hypothesis A. The sequence $\left\{\alpha_{j}\right\}$ of probabilities (introduced in Theorem 10) satisfies the conditions: $0<\alpha_{j}<1(j \geq 0),\left\{\alpha_{j}\right\}$ is monotonic and decreasing from some point onward (i.e. for $\left.j \geq j_{1}\right)$, and $\alpha_{j} \rightarrow 0$ as $j \rightarrow \infty$.

We have the following result for $s_{N}^{*}(\omega)$ and its expected value $m_{N}^{*}$.

Lemma 12. If, in addition to Hypothesis $A$,

$$
m_{N}^{*} \rightarrow \infty
$$

as $N \rightarrow \infty$, and

$$
\sum_{N=0}^{\infty} \frac{\alpha_{N}}{\left(m_{N}^{*}\right)^{2}}<\infty
$$

then with probability 1 , we have $s_{N}^{*}(\omega) \sim m_{N}^{*}$ as $N \rightarrow \infty$. 
Proof. We denote $D^{2}(f)$ to be the variance of a random variable $f$, defined by

$$
D^{2}(f)=E\left((f-E(f))^{2}\right) .
$$

The proof is basically an application of a variant of the strong law of large numbers [3, p. 140, Theorem 11], which is as follows. Let $\left\{f_{j}\right\}$ be a sequence of independent random variables, and let

$$
s_{i}(\omega)=\sum_{j=0}^{i} f_{j}(\omega) \quad(i \geq 0) .
$$

Suppose we have

$$
\begin{gathered}
E\left(f_{j}\right)>0 \quad(j \geq 0), \\
\lim _{i \rightarrow \infty} E\left(s_{i}\right)=\infty,
\end{gathered}
$$

and

$$
\sum_{i=0}^{\infty} \frac{D^{2}\left(f_{i}\right)}{\left(E\left(s_{i}\right)\right)^{2}}<\infty
$$

Then, with probability 1 , we have

$$
s_{i}(\omega)=(1+\mathbf{o}(1)) E\left(s_{i}\right)
$$

as $i \rightarrow \infty$. We know that the sets $\mathfrak{B}_{j}$ are independent, which is equivalent to $\mathbf{1}_{\mathfrak{B}_{j}}(\omega)$ being independent. Thus we apply this theorem with $f_{j}(\omega)=\mathbf{1}_{\mathfrak{B}_{j}}(\omega)$, and obtain our result.

For every $N, d \in \mathbb{Z}_{\geq 0}$, we define the event $\mathfrak{e}(N, d)$ as

$$
\mathfrak{e}(N, d)=\left\{\omega \in \Omega: r_{N}(\omega)=d\right\} .
$$

As mentioned in Section 1, we need to study the probability of the event $\mathfrak{e}(N, d)$. We start with the following lemma.

Lemma 15. For all non-negative integers $N$ and $d$, we have

$$
P(\mathfrak{e}(N, d))=\left(\prod_{1 \leq k \leq M}\left(1-\alpha_{a_{k}} \alpha_{\widetilde{a}_{k}}\right)\right) \widetilde{\sigma}_{d}(N),
$$

where $\widetilde{\sigma}_{0}(N)=1$ and, if $d \geq 1$,

$$
\widetilde{\sigma}_{d}(N)=\sum_{1 \leq k_{1}<\ldots<k_{d} \leq M} \prod_{1 \leq i \leq d} \frac{\alpha_{a_{k_{i}}} \alpha_{\widetilde{a}_{k_{i}}}}{1-\alpha_{a_{k_{i}}} \alpha_{\widetilde{a}_{k_{i}}}} .
$$

Proof. We begin with the case $d=0$. It is easy to see that

$$
\mathfrak{e}(N, 0)=\bigcap_{1 \leq k \leq M}\left(\mathfrak{B}_{a_{k}} \cap \mathfrak{B}_{\widetilde{a}_{k}}\right)^{\mathbf{c}},
$$

where $\mathbf{c}$ denotes taking the complement of the set. Since the sets $\mathfrak{B}_{j}(j \geq 0)$ are independent, we know that $\mathfrak{B}_{a_{k}} \cap \mathfrak{B}_{\widetilde{a}_{k}}(1 \leq k \leq M)$ are independent as $\left\{a_{k}: 1 \leq k \leq M\right\} \cap\left\{\widetilde{a}_{k}: 1 \leq k \leq M\right\}=\varnothing$. Thus, it follows that $\left(\mathfrak{B}_{a_{k}} \cap \mathfrak{B}_{\widetilde{a}_{k}}\right)^{\mathbf{c}}(1 \leq k \leq M)$ are also independent. Hence, we have

$$
P(\mathfrak{e}(N, 0))=\prod_{1 \leq k \leq M} P\left(\left(\mathfrak{B}_{a_{k}} \cap \mathfrak{B}_{\widetilde{a}_{k}}\right)^{\mathbf{c}}\right)=\prod_{1 \leq k \leq M}\left(1-\alpha_{a_{k}} \alpha_{\widetilde{a}_{k}}\right) .
$$


Suppose $1 \leq d \leq M$ and $\omega^{\prime} \in \mathfrak{e}(N, d)$. Then there exist $k_{1}, k_{2}, \ldots, k_{d}$ such that $1 \leq k_{i} \leq M$, $a_{k_{i}}, \widetilde{a}_{k_{i}} \in \omega^{\prime}(1 \leq i \leq d)$, and further, if $k \neq k_{i}$ and $1 \leq k \leq M$, then we have either $a_{k} \notin \omega^{\prime}$ or $\widetilde{a}_{k} \notin \omega^{\prime}$. From this observation, we can deduce that

$$
P(\mathfrak{e}(N, d))=\sum_{1 \leq k_{1}<\ldots<k_{d} \leq M} P\left(\mathfrak{E}\left(k_{1}, \ldots, k_{d}\right)\right),
$$

where $\mathfrak{E}\left(k_{1}, \ldots, k_{d}\right)$ is the event

$$
\bigcap_{1 \leq i \leq d}\left(\mathfrak{B}_{a_{k_{i}}} \cap \mathfrak{B}_{\widetilde{a}_{k_{i}}}\right) \bigcap \bigcap_{\substack{1 \leq k \leq M \\ k \neq k_{i}(1 \leq i \leq d)}}\left(\mathfrak{B}_{a_{k}} \cap \mathfrak{B}_{\widetilde{a}_{k}}\right)^{\mathbf{c}} .
$$

Again, by independence, we have

$$
\begin{aligned}
P\left(\mathfrak{E}\left(k_{1}, \ldots, k_{d}\right)\right) & =\prod_{1 \leq i \leq d} P\left(\mathfrak{B}_{a_{k_{i}}} \cap \mathfrak{B}_{\widetilde{a}_{k_{i}}}\right) \cdot \prod_{\substack{1 \leq k \leq M \\
k \neq k_{i}(1 \leq i \leq d)}} P\left(\left(\mathfrak{B}_{a_{k}} \cap \mathfrak{B}_{\widetilde{a}_{k}}\right)^{\mathbf{c}}\right) \\
& =\prod_{1 \leq i \leq d} \alpha_{a_{k_{i}}} \alpha_{\widetilde{a}_{k_{i}}} \cdot \prod_{\substack{1 \leq k \leq M \\
k \neq k_{i}(1 \leq i \leq d)}}\left(1-\alpha_{a_{k}} \alpha_{\widetilde{a}_{k}}\right) \\
& =\prod_{1 \leq k \leq M}\left(1-\alpha_{a_{k}} \alpha_{\widetilde{a}_{k}}\right) \cdot \prod_{1 \leq i \leq d} \frac{\alpha_{a_{k_{i}}} \alpha_{\widetilde{a}_{k_{i}}}}{1-\alpha_{a_{k_{i}}} \alpha_{\widetilde{a}_{k_{i}}}}
\end{aligned}
$$

from which the desired result follows.

Finally, if $d>M$, then the sum $\widetilde{\sigma}_{d}(N)$ is empty, and both sides of (16) are 0 .

To estimate $\widetilde{\sigma}_{d}(N)$, we use the following result for elementary symmetric functions.

Lemma 18. [3, p. 147, Lemma 13] Let $y_{1}, y_{2}, \ldots y_{M^{\prime}}$ be $M^{\prime}$ non-negative real numbers. For each positive integer $d$, not exceeding $M^{\prime}$, let

$$
\sigma_{d}=\sum_{1 \leq k_{1}<\ldots<k_{d} \leq M^{\prime}} y_{k_{1}} y_{k_{2}} \ldots y_{k_{d}},
$$

so that $\sigma_{d}$ is the $d$-th elementary symmetric function of the $y_{k}$ 's. Then, for each $d$, we have

$$
\frac{1}{d !} \sigma_{1}^{d}\left(1-\left(\begin{array}{l}
d \\
2
\end{array}\right) \frac{1}{\sigma_{1}^{2}} \sum_{k=1}^{M^{\prime}} y_{k}^{2}\right) \leq \sigma_{d} \leq \frac{1}{d !} \sigma_{1}^{d},
$$

where we interpret $\left(\begin{array}{l}d \\ 2\end{array}\right)$ to be 0 when $d=1$.

The next lemma gives us bounds on the probability of the event $\mathfrak{e}(N, d)$ in terms of $\lambda_{N}$ and $\lambda_{N}^{\prime}$.

Lemma 20. Let $N$ and $d$ be non-negative integers. Then we have

$$
P(\mathfrak{e}(N, d)) \leq \frac{\left(\lambda_{N}^{\prime}\right)^{d}}{d !} e^{-\lambda_{N}} .
$$

Furthermore, if $d \leq M$, we have

$$
P(\mathfrak{e}(N, d)) \geq \frac{\left(\lambda_{N}^{\prime}\right)^{d}}{d !} e^{-\lambda_{N}^{\prime}}\left(1-\left(\begin{array}{l}
d \\
2
\end{array}\right)\left(\lambda_{N}^{\prime}\right)^{-2} Q^{*}\right),
$$


where

$$
Q^{*}=\sum_{1 \leq k \leq M}\left(\frac{\alpha_{a_{k}} \alpha_{\widetilde{a}_{k}}}{1-\alpha_{a_{k}} \alpha_{\widetilde{a}_{k}}}\right)^{2}
$$

and $\left(\begin{array}{l}d \\ 2\end{array}\right)$ is interpreted to be 0 if $d<2$.

ProOF. We note that if $d>M$, then the event $\mathfrak{e}(N, d)$ is empty and (21) is trivial. Suppose $1 \leq d \leq M$. We apply (19) with $M^{\prime}=M$ and $y_{k}=\alpha_{a_{k}} \alpha_{\widetilde{a}_{k}} /\left(1-\alpha_{a_{k}} \alpha_{\widetilde{a}_{k}}\right)$ to estimate $\widetilde{\sigma}_{d}(N)$ in $(16)$; thus noting that $\widetilde{\sigma}_{1}(N)=\lambda_{N}^{\prime}$, we obtain

$$
P(\mathfrak{e}(N, d)) \leq\left(\prod_{1 \leq k \leq M}\left(1-\alpha_{a_{k}} \alpha_{\widetilde{a}_{k}}\right)\right) \frac{\left(\lambda_{N}^{\prime}\right)^{d}}{d !}
$$

and

$$
P(\mathfrak{e}(N, d)) \geq\left(\prod_{1 \leq k \leq M}\left(1-\alpha_{a_{k}} \alpha_{\widetilde{a}_{k}}\right)\right) \frac{\left(\lambda_{N}^{\prime}\right)^{d}}{d !}\left(1-\left(\begin{array}{l}
d \\
2
\end{array}\right)\left(\lambda_{N}^{\prime}\right)^{-2} Q^{*}\right) .
$$

Applying the inequality $e^{-t /(1-t)}<1-t<e^{-t}$, which holds for $0<t<1$, with $t=\alpha_{a_{k}} \alpha_{\widetilde{a}_{k}}(1 \leq$ $k \leq M)$, we obtain

$$
e^{-\lambda_{N}^{\prime}}<\left(\prod_{1 \leq k \leq M}\left(1-\alpha_{a_{k}} \alpha_{\widetilde{a}_{k}}\right)\right)<e^{-\lambda_{N}}
$$

and our result follows. When $d=0$, we have

$$
P(\mathfrak{e}(N, d))=\prod_{1 \leq k \leq M}\left(1-\alpha_{a_{k}} \alpha_{\widetilde{a}_{k}}\right),
$$

and the result is immediate from (23) in this case.

To estimate $\lambda_{N}$ and $\lambda_{N}^{\prime}$, we first prove the following lemma.

Lemma 24. If Hypothesis $A$ is satisfied, then

$$
\lambda_{N}^{\prime} \sim \lambda_{N}
$$

as $N \rightarrow \infty$.

Proof. Recall from (11) that if $1 \leq k \leq M$, then $q^{n} \leq \widetilde{a}_{k}<q^{n+1}$. Consequently, we have

$$
\alpha_{a_{k}} \alpha_{\widetilde{a}_{k}}<\alpha_{\widetilde{a}_{k}} \leq \alpha_{q^{n}}=\mathbf{o}(1)
$$

as $N \rightarrow \infty$. Therefore, we obtain

$$
\begin{aligned}
\lambda_{N}^{\prime}-\lambda_{N} & =\sum_{1 \leq k \leq M} \alpha_{a_{k}} \alpha_{\widetilde{a}_{k}}\left(\frac{1}{1-\alpha_{a_{k}} \alpha_{\widetilde{a}_{k}}}-1\right) \\
& =\sum_{1 \leq k \leq M} \alpha_{a_{k}} \alpha_{\widetilde{a}_{k}}\left(\frac{\alpha_{a_{k}} \alpha_{\widetilde{a}_{k}}}{1-\alpha_{a_{k}} \alpha_{\widetilde{a}_{k}}}\right) \\
& \leq \alpha_{q^{n}} \lambda_{N}^{\prime}
\end{aligned}
$$

from which the result follows. 
Therefore, it is enough to estimate $\lambda_{N}$. The following lemma gives us an estimate for $\lambda_{N}$ sufficient for our purpose.

Lemma 26. Suppose that the sequence $\left\{\alpha_{j}\right\}$, introduced in Theorem 10, is such that

$$
\alpha_{j}=\alpha \frac{(\log j)^{c^{\prime}}}{j^{c}}
$$

for $j \geq j_{0}$; where $j_{0}, \alpha, c, c^{\prime}$ are constants such that $\alpha>0,0<\alpha_{j}<1(j \geq 0), 0<c<1$, and $c^{\prime} \geq 0$. Then, for sufficiently large $H$, there exist positive constants $D_{1}$ and $D_{2}$, which depend at most on $c, c^{\prime}$, and $q$, such that

$$
\alpha^{2} D_{1}(\log N)^{2 c^{\prime}} q^{n(1-2 c)}<\lambda_{N}<\alpha^{2} D_{2}(\log N)^{2 c^{\prime}} q^{n(1-2 c)}
$$

for all $N>H$.

Furthermore, we have

$$
m_{N}^{*} \sim \frac{\alpha}{1-c}(\log N)^{c^{\prime}} N^{(1-c)} .
$$

Finally, if $c^{\prime}=0$, then with probability 1 , the numbers $b_{j}$ of the sequence $\omega=\left\{p_{b_{j}}\right\}$ satisfy

$$
b_{j} \sim\left(\frac{1-c}{\alpha} j\right)^{1 /(1-c)}
$$

as $j \rightarrow \infty$.

Proof. We begin by finding a lower bound for $\lambda_{N}$. We assume $p \neq 2$. The case $p=2$ can be treated in a similar manner. Suppose $N>q \cdot\left(j_{0}+1\right)$ from which it follows that $q^{n}>j_{0}$. Let $C_{0}$ and $C_{0}^{\prime}$ be the positive constants defined by

$$
C_{0}=\sum_{1 \leq j<j_{0}} \alpha_{j}
$$

and

$$
C_{0}^{\prime}=\sum_{1 \leq j<j_{0}} \frac{(\log j)^{c^{\prime}}}{j^{c}} .
$$

Since $q^{n} \leq \widetilde{a}_{i}<q^{n+1}, 0 \leq a_{i}<q^{n+1}$, and $(\log x)^{c^{\prime}} / x^{c}$ is a decreasing function, we obtain

$$
\begin{aligned}
\lambda_{N}=\sum_{1 \leq i \leq M} \alpha_{a_{i}} \alpha_{\widetilde{a}_{i}} & >\frac{\alpha\left(\log q^{n+1}\right)^{c^{\prime}}}{q^{(n+1) c}} \sum_{1 \leq i \leq M} \alpha_{a_{i}} \\
& >\frac{\alpha^{2}\left(\log q^{n+1}\right)^{c^{\prime}}}{q^{(n+1) c}}\left(\sum_{j=\left(q^{n+1}-1\right) / 2}^{q^{n+1}-1} \frac{(\log j)^{c^{\prime}}}{j^{c}}-C_{0}^{\prime}\right) \\
& >\frac{\alpha^{2}\left(\log q^{n}\right)^{2 c^{\prime}}}{q^{(n+1) c}}\left(\sum_{j=\left(q^{n+1}-1\right) / 2}^{q^{n+1}-1} \frac{1}{j^{c}}-C_{0}^{\prime}\right) .
\end{aligned}
$$

We know that for all $s, t \in \mathbb{N}, 0<s<t$,

$$
\frac{1}{1-c}(t+1)^{1-c}-\frac{1}{1-c} s^{1-c} \leq \sum_{s \leq j \leq t} \frac{1}{j^{c}} \leq \frac{1}{1-c} t^{1-c}-\frac{1}{1-c}(s-1)^{1-c} .
$$


Thus, by (30) we can give the following lower bound for $\lambda_{N}$,

$$
\begin{aligned}
\lambda_{N} & >\frac{\alpha^{2}\left(\log q^{n}\right)^{2 c^{\prime}}}{q^{(n+1) c}}\left(\frac{1}{1-c}\left(q^{n+1}\right)^{1-c}-\frac{1}{1-c}\left(\frac{q^{n+1}-1}{2}\right)^{1-c}-C_{0}^{\prime}\right) \\
& =\frac{\alpha^{2}\left(\log q^{n}\right)^{2 c^{\prime}}}{(1-c) q^{c}} q^{n(1-2 c)}\left(q^{1-c}-\left(\frac{q}{2}-\frac{1}{2 q^{n}}\right)^{1-c}-\frac{1-c}{q^{n(1-c)}} \cdot C_{0}^{\prime}\right) .
\end{aligned}
$$

Since $q^{n} \leq N<q^{n+1}$, we have $\log N(1-\log q / \log N)<\log q^{n}$. It follows from (31) that by taking $H$ sufficiently large, we obtain

$$
\lambda_{N}>\alpha^{2} \frac{q^{1-2 c}}{2(1-c)}\left(1-\frac{1}{2^{1-c}}\right)(\log N)^{2 c^{\prime}} q^{n(1-2 c)}
$$

for all $N>H$.

Next, we would like to find an upper bound for $\lambda_{N}$. Again, since $q^{n} \leq \widetilde{a}_{i}<q^{n+1}$ and $0 \leq a_{i}<$ $q^{n+1}$, by similar calculations as before we have

$$
\lambda_{N}<\frac{\alpha\left(\log q^{n+1}\right)^{c^{\prime}}}{q^{n c}} \sum_{1 \leq i \leq M} \alpha_{a_{i}}<\frac{\alpha\left(\log q^{n+1}\right)^{c^{\prime}}}{q^{n c}}\left(C_{0}+\alpha \sum_{j=1}^{M} \frac{\left(\log q^{n+1}\right)^{c^{\prime}}}{j^{c}}\right) .
$$

Thus, by applying (30), we obtain

$$
\begin{aligned}
\lambda_{N} & <\frac{\alpha^{2}\left(\log q^{n+1}\right)^{2 c^{\prime}}}{q^{n c}}\left(\frac{C_{0}}{\alpha\left(\log q^{n+1}\right)^{c^{\prime}}}+\frac{1}{1-c}\left(\frac{q^{n+1}-1}{2}\right)^{1-c}\right) \\
& =\frac{\alpha^{2}\left(\log q^{n+1}\right)^{2 c^{\prime}}}{(1-c)} q^{n(1-2 c)}\left(\frac{C_{0}(1-c)}{\alpha\left(\log q^{n+1}\right)^{c^{\prime}} q^{n(1-c)}}+\left(\frac{q}{2}-\frac{1}{2 q^{n}}\right)^{1-c}\right) .
\end{aligned}
$$

Therefore, by taking $H$ sufficiently large, we obtain that

$$
\lambda_{N}<\alpha^{2} \frac{2^{2 c^{\prime}+1}}{(1-c)}\left(\frac{q}{2}\right)^{1-c}\left(\log q^{n}\right)^{2 c^{\prime}} q^{n(1-2 c)}
$$

for all $N>H$. Then, since $q^{n} \leq N<q^{n+1}$, we are done with the first part of the lemma.

Clearly, we have

$$
m_{N}^{*}=\sum_{j=1}^{N} \alpha \frac{(\log j)^{c^{\prime}}}{j^{c}}+\mathbf{O}(1)=(1+\mathbf{o}(1)) \frac{\alpha}{1-c}(\log N)^{c^{\prime}} N^{(1-c)},
$$

and this proves (28). We note (28) shows that (13) and (14) are satisfied.

The final assertion of the lemma follows from (28), in view of Lemma 12, and the fact that $s_{b_{j}}^{*}(\omega)=j$ for $\omega=\left\{p_{b_{j}}\right\}_{j \in \mathbb{N}}$; for in this way it follows that, if $c^{\prime}=0$, we have with probability 1 ,

$$
j=s_{b_{j}}^{*}(\omega) \sim m_{b_{j}}^{*} \sim \frac{\alpha}{1-c} b_{j}^{1-c},
$$

or equivalently, $b_{j} \sim\left(\frac{1-c}{\alpha} j\right)^{1 /(1-c)}$. 
We also make use of the following lemma.

Lemma 33. [3, p. 149, Lemma 17] If $0<\xi \leq U$, then

$$
\sum_{d \geq U} \frac{\xi^{d}}{d !} \leq\left(\frac{e \xi}{U}\right)^{U}
$$

and if $0<V \leq \xi$, then

$$
\sum_{0 \leq d \leq V} \frac{\xi^{d}}{d !} \leq\left(\frac{e \xi}{V}\right)^{V}
$$

\section{Proof of Theorem 7}

Let $c=c^{\prime}=1 / 2$. We choose a number $\alpha>0$ to satisfy

$$
\alpha^{2} \frac{q^{1-2 c}}{2(1-c)}\left(1-\frac{1}{2^{1-c}}\right)>1 .
$$

We then define a sequence $\left\{\alpha_{j}\right\}$ by

$$
\alpha_{j}=\alpha\left(\frac{\log j}{j}\right)^{1 / 2}
$$

for all $j \geq j_{0}$, where $j_{0}$ is a positive integer sufficient large such that the expression in (34) is less than $1 / 2$ for all $j \geq j_{0}$. For $1 \leq j<j_{0}$, we let $\alpha_{j}=1 / 2$. The precise value of $\alpha_{j}$ for small $j$ is unimportant, but the above choices ensure $0<\alpha_{j}<1$, so that Hypothesis A is satisfied. By (32) in the proof of Lemma 26, we have for all $N$ sufficiently large that

$$
\lambda_{N} \geq \alpha^{2} D_{1} \log N>\log N .
$$

Hence, we know there exists $\delta>0$ such that

$$
e^{-\lambda_{N}} \ll N^{-1-\delta} .
$$

We establish the theorem by showing that, with probability $1, \log N \ll r_{N}(\omega) \ll \log N$ for large $N$, or equivalently (in view of Lemmas 24 and 26)

$$
\lambda_{N}^{\prime} \ll r_{N}(\omega) \ll \lambda_{N}^{\prime}
$$

for $N>N_{0}(\omega)$. We apply the Borel-Cantelli lemma twice to prove that each of the two assertions of (37) holds with probability 1 . For this purpose, we must show that if $C_{1}, C_{2}$ are suitably chosen positive constants, then we have

$$
\sum_{N=0}^{\infty} P\left(\left\{\omega \in \Omega: r_{N}(\omega)>C_{1} \lambda_{N}^{\prime}\right\}\right)<\infty
$$

and

$$
\sum_{N=0}^{\infty} P\left(\left\{\omega \in \Omega: r_{N}(\omega)<C_{2} \lambda_{N}^{\prime}\right\}\right)<\infty .
$$


By Lemmas 20 and 33, we have

$$
\begin{aligned}
P\left(\left\{\omega \in \Omega: r_{N}(\omega)>C_{1} \lambda_{N}^{\prime}\right\}\right) \leq & e^{-\lambda_{N}} \sum_{d \geq C_{1} \lambda_{N}^{\prime}} \frac{\left(\lambda_{N}^{\prime}\right)^{d}}{d !} \\
& \leq e^{-\lambda_{N}}\left(\frac{e}{C_{1}}\right)^{C_{1} \lambda_{N}^{\prime}},
\end{aligned}
$$

provided $C_{1} \geq 1$. Thus, by choosing $C_{1}=e$, we obtain a bound $e^{-\lambda_{N}}$ for the summand of (38), and the inequality (38) follows from (36).

On the other hand, again by Lemmas 20 and 33, we obtain the following estimate for the summand of (39),

$$
\begin{array}{r}
P\left(\left\{\omega \in \Omega: r_{N}(\omega)<C_{2} \lambda_{N}^{\prime}\right\}\right) \leq e^{-\lambda_{N}} \sum_{0 \leq d \leq C_{2} \lambda_{N}^{\prime}} \frac{\left(\lambda_{N}^{\prime}\right)^{d}}{d !} \\
\leq e^{-\lambda_{N}}\left(\frac{e}{C_{2}}\right)^{C_{2} \lambda_{N}^{\prime}},
\end{array}
$$

provided $C_{2} \leq 1$. Thus, it suffices to show that $C_{2}$ can be chosen to satisfy, in addition to $0<C_{2} \leq 1$,

$$
\left(\frac{e}{C_{2}}\right)^{C_{2} \lambda_{N}^{\prime}} \ll N^{\delta / 2} ;
$$

for (39) will then follow from (36). By Lemmas 24 and 26, we know there exists $D>0$ such that $\lambda_{N}^{\prime} \leq D \log N$ for $N$ sufficiently large. Therefore, we only need to choose a small positive constant $C_{2}$ satisfying

$$
\left(\frac{e}{C_{2}}\right)^{C_{2}} \leq e^{\delta /(2 D)}
$$

which is certainly possible since $(e / t)^{t} \rightarrow 1$ as $t \rightarrow 0$ from the positive side.

We have now shown that $\omega$ has each of the desired properties with probability 1 , and this proves the theorem.

\section{Proof of Theorem 8}

Let $\epsilon>0$ be given. We define a sequence $\left\{\alpha_{j}\right\}$ by $\alpha_{0}=1 / 2$ and

$$
\alpha_{j}=\frac{1}{2 j^{1-1 /(2+\epsilon)}}
$$

for $j \geq 1$. It then follows by Lemma 26 (with $\alpha=1 / 2, c=1-1 /\left(2+\epsilon\right.$ ), and $c^{\prime}=0$ ) that, with probability $1, \omega=\left\{p_{b_{j}}\right\}$ satisfies $b_{j} \sim c^{*} j^{2+\epsilon}$, where $c^{*}$ is some positive constant.

Since the sequence $\left\{\alpha_{j}\right\}$ satisfies Hypothesis A, we have $\lambda_{N}^{\prime} \sim \lambda_{N}$ by Lemma 24. Thus, by Lemma 26 we know that there exist positive constants $D_{1}$ and $D_{2}$ such that

$$
D_{1} q^{-\epsilon n /(2+\epsilon)}<\lambda_{N}, \lambda_{N}^{\prime}<D_{2} q^{-\epsilon n /(2+\epsilon)}
$$

for $N$ sufficiently large. 
We again appeal to the Borel-Cantelli Lemma. It follows from this lemma that if a positive number $K$ satisfies the property

$$
\sum_{N=0}^{\infty} P\left(\left\{\omega \in \Omega: r_{N}(\omega) \geq K\right\}\right)<\infty,
$$

then, with probability 1 , we have

$$
r_{N}(\omega)<K
$$

for $N>N_{0}(\omega)$.

We note that, by (40), $\lambda_{N} \rightarrow 0$ and $\lambda_{N}^{\prime} \rightarrow 0$ as $N \rightarrow \infty$. Thus, by Lemmas 20 and 33, we obtain the following estimate for the summand of (41),

$$
P\left(\left\{\omega \in \Omega: r_{N}(\omega) \geq K\right\}\right) \leq e^{-\lambda_{N}} \sum_{d \geq K} \frac{\left(\lambda_{N}^{\prime}\right)^{d}}{d !} \leq e^{-\lambda_{N}}\left(\frac{e \lambda_{N}^{\prime}}{K}\right)^{K} \ll\left(\lambda_{N}^{\prime}\right)^{K}
$$

for $N$ sufficiently large. Since $q^{n} \leq N<q^{n+1}$, we have

$$
\left(\lambda_{N}^{\prime}\right)^{K} \leq D_{2}^{K} q^{-\epsilon n K /(2+\epsilon)} \ll N^{-\epsilon K /(2+\epsilon)} .
$$

Therefore, provided $\epsilon K /(2+\epsilon)>1$, or equivalently,

$$
K>1+2 \epsilon^{-1},
$$

it is clear that (41) is achieved. Accordingly we have, with probability 1 ,

$$
r_{N}(\omega)<2\left(1+\epsilon^{-1}\right)
$$

for $N>N_{1}(\epsilon, \omega)$. This completes the proof of the theorem.

\section{Proof of Theorem 9}

Recall we defined $t_{N}(\omega)$ to be

$$
t_{N}(\omega)=\left|\left\{(a, b) \in \mathbb{Z}_{\geq 0} \times \mathbb{Z}_{\geq 0}: p_{a}, p_{b} \in \omega, p_{N}=p_{a}-p_{b}, \operatorname{deg} p_{a}, \operatorname{deg} p_{b} \leq \operatorname{deg} p_{N}\right\}\right| .
$$

As before given $p_{N} \in \mathbb{F}_{q}[T]$, we let $n:=n(N)=\operatorname{deg} p_{N}=\left\lfloor\log _{q} N\right\rfloor$. It is clear that for $p_{N} \neq 0$, there exist $q^{n+1}$ pairs of polynomials $\left(p_{a}, p_{b}\right)$ such that $p_{N}=p_{a}-p_{b}$ and $\operatorname{deg} p_{a}, \operatorname{deg} p_{b} \leq n$. Also, every polynomial of degree less than or equal to $n$ will appear as $p_{a}$ and $p_{b}$ exactly once. Let $S_{\widehat{u}, n}$ denote the set of all polynomials in $\mathbb{F}_{q}[T]$ whose degree are less than or equal to $n$, and the coefficient of $T^{n}$ is $\widehat{u} \in \mathbb{F}_{q}$. Clearly, we have $\left|S_{\widehat{u}, n}\right|=q^{n}$. If we consider each polynomial in $S_{\widehat{u}, n}$ as $p_{b}$, then the corresponding set of $p_{a}$ 's is $S_{u, n}$ for some $u \neq \widehat{u}$ as $\operatorname{deg} p_{N}=n$.

For each $u \in \mathbb{F}_{q}$, we consider

$$
t_{N, u}(\omega)=\mid\left\{(a, b) \in \mathbb{Z}_{\geq 0} \times \mathbb{Z}_{\geq 0}: p_{N}=p_{a}-p_{b} \text {, where } p_{a}, p_{b} \in \omega \text { and } p_{a} \in S_{u, n}\right\} \mid .
$$

If $p_{N}=p_{a}-p_{b}$, we relabel $p_{b}$ as $p_{\widehat{a}}$ to make its correspondence with $p_{a}$ more explicit. We form the following two disjoint sets

$$
\mathcal{A}_{N}=\left\{a \in \mathbb{Z}_{\geq 0}: p_{a} \in S_{u, n}\right\}=\left\{\iota^{-1}(u) q^{n}, \ldots,\left(\iota^{-1}(u)+1\right) q^{n}-1\right\}
$$

and

$$
\widehat{\mathcal{A}}_{N}=\left\{\widehat{a} \in \mathbb{Z}_{\geq 0}: p_{\widehat{a}} \in S_{\widehat{u}, n}\right\}=\left\{\iota^{-1}(\widehat{u}) q^{n}, \ldots,\left(\iota^{-1}(\widehat{u})+1\right) q^{n}-1\right\} .
$$

Let $M_{0}:=M_{0}(N)=\left|\mathcal{A}_{N}\right|=\left|\widehat{\mathcal{A}}_{N}\right|=q^{n}$. For convenience, we label the $M_{0}$ elements of $\mathcal{A}_{N}$ by $a_{i}\left(1 \leq i \leq M_{0}\right)$, and the corresponding elements of $\widehat{\mathcal{A}}_{N}$ by $\widehat{a}_{i}$, in other words we have $p_{N}=$ $p_{a_{i}}-p_{\widehat{a}_{i}}\left(1 \leq i \leq M_{0}\right)$. 
We also define $\lambda_{N, u}$ and $\lambda_{N, u}^{\prime}$ to be

$$
\lambda_{N, u}=\sum_{1 \leq i \leq M_{0}} \alpha_{a_{i}} \alpha_{\widehat{a}_{i}}
$$

and

$$
\lambda_{N, u}^{\prime}=\sum_{1 \leq i \leq M_{0}} \frac{\alpha_{a_{i}} \alpha_{\widehat{a}_{i}}}{1-\alpha_{a_{i}} \alpha_{\widehat{a}_{i}}} .
$$

With this set up we can recover analogues of all the previous lemmas in terms of $M_{0}, \lambda_{N, u}, \lambda_{N, u}^{\prime}$, and $t_{N, u}(\omega)$, in place of $M, \lambda_{N}, \lambda_{N}^{\prime}$, and $r_{N}(\omega)$, respectively. Therefore, by a similar argument we obtain Theorem 8 with $t_{N, u}(\omega)$ in place of $r_{N}(\omega)$. Since this result holds with probability 1 , and

$$
t_{N}(\omega)=\sum_{u \in \mathbb{F}_{q}} t_{N, u}(\omega)
$$

we have our result.

\section{REFERENCES}

[1] P. Erdős, On a problem of Sidon in additive number thoery, Acta Sci. Math. Szeged 15 (1954), pp255-259.

[2] P. Erdős and A. Rényi, Additive properties of random sequences of positive integers, Acta Arith. 6 (1960), 83-110.

[3] H. Halberstam and K.F. Roth, Sequences, Springer-Verlag, New York, 1983.

[4] K. E. Hare and S. Yamagishi, A generalization of a theorem of Erdös-Rényi to $m$-fold sums and differences, to appear in Acta Arithmetica.

Department of Pure Mathematics, University of Waterloo, Waterloo, on, N2L 3G1, Canada

E-mail address: wtkuo@uwaterloo.ca

Department of Pure Mathematics, University of Waterloo, Waterloo, On, N2L 3G1, Canada

E-mail address: syamagis@uwaterloo.ca 